\title{
Approximation exponents for algebraic functions in positive characteristic
}

\author{
by
}

\author{
Bernard de Mathan (Talence)
}

In this paper, we study rational approximations for algebraic functions in characteristic $p>0$. We obtain results for elements satisfying an equation of the type $\alpha=\left(A \alpha^{q}+B\right) /\left(C \alpha^{q}+D\right)$, where $q$ is a power of $p$.

1. Introduction and notations. Let $K$ be a field, and let $K\left(\left(T^{-1}\right)\right)$ be the field of formal Laurent series in $1 / T$. For $f \in K\left(\left(T^{-1}\right)\right), \operatorname{deg}(f)$ is the integer defined by $f=\sum_{n=-\infty}^{\operatorname{deg}(f)} a_{n} T^{n}$, with $a_{\operatorname{deg}(f)} \neq 0$. We define an absolute value on $K\left(\left(T^{-1}\right)\right)$ by $|f|=|T|^{\operatorname{deg}(f)}$, where $|T|>1$. For each $f \in K\left(\left(T^{-1}\right)\right)$, there exists a polynomial $E(f)$ in $K[T]$ (integral part of $f$ ) such that $|f-E(f)|<1$. We denote $|f-E(f)|$ by $\|f\|$.

Let $\alpha \in K\left(\left(T^{-1}\right)\right)$. For any real number $\mu$, define

$$
B(\alpha, \mu)=\liminf _{|Q| \rightarrow \infty}|Q|^{\mu}\|Q \alpha\|
$$

We define the approximation exponent of $\alpha$ by

$$
\nu(\alpha)=\sup \{\mu \mid B(\alpha, \mu)<\infty\} .
$$

Clearly $B(\alpha, 1) \leq 1 /|T|$, hence $\nu(\alpha) \geq 1$ for every $\alpha$. Let $\left(Q_{n}\right)_{n \in \mathbb{N}}$ be the sequence of the denominators of the convergents in the continued fraction expansion of $\alpha$. One has

$$
\nu(\alpha)=\lim \sup \operatorname{deg}\left(Q_{n+1}\right) / \operatorname{deg}\left(Q_{n}\right) .
$$

It is easy to see that $\nu(\alpha)$ may be any real number $\nu \geq 1$ or $\nu=+\infty$.

It is well known that if $K$ has characteristic 0, Roth's Theorem remains valid ([7]), i.e. $\nu(\alpha)=1$ for every algebraic irrational element $\alpha$ of $K\left(\left(T^{-1}\right)\right)$. On the other hand, if $K$ has a positive characteristic, $p$, Roth's Theorem fails. The Liouville Theorem holds, i.e. $\nu(\alpha) \leq n-1$ if $\alpha$ is algebraic, of degree $n>1$, over $K(T)$. But this result is the best possible, as many examples show. For instance, let $q$ be a power of $p$, and $q>2$. Let $\alpha=$ $T^{-1}+\ldots+T^{-q^{k}}+\ldots$; this element satisfies the equation $\alpha^{q}-\alpha+T^{-1}=$ 
0 , and $\nu(\alpha)=q-1$ (Mahler's example). Osgood's example is $\alpha$ such that $\alpha^{q-1}=1+T^{-1}$, for which $\nu(\alpha)=q-2(q>3)$. One can also cite $\alpha = 1 \sqrt { T } + 1 \sqrt { T ^ { q } } + \ldots + 1 \longdiv { T ^ { q ^ { k } } } + \ldots$; this element $\alpha$ satisfies $\alpha^{q+1}+T \alpha-1=0$, and $\nu(\alpha)=q($ for $q \geq 2)$.

Nevertheless, there exist in $K\left(\left(T^{-1}\right)\right)$ algebraic elements $\alpha$ of degrees $>2$ for which $\nu(\alpha)=1$. The first example was obtained by Baum and Sweet $([1])$ : in $\mathbb{F}_{2}\left(\left(T^{-1}\right)\right), \alpha$ such that $\alpha^{3}+T^{-1} \alpha+1=0$ is a cubic element for which $\nu(\alpha)=1$ (and $B(\alpha, 1)=|T|^{-2}$ ). Other examples were given by W. H. Mills and D. P. Robbins ([4]), for other characteristics. Examples of algebraic elements $\alpha$ such that $1<\nu(\alpha)<d(\alpha)-1$, where $d(\alpha)$ is the degree of $\alpha$ over $K(T)$, were also found by Y. Taussat ([6]): for $\alpha \in \mathbb{F}_{3}\left(\left(T^{-1}\right)\right)$ such that $\alpha^{4}+T^{-1} \alpha-1=0$, one has $\nu(\alpha)=23 / 19\left(\right.$ and $B(\alpha, \nu(\alpha))=|T|^{-21 / 19}$, $d(\alpha)=4)$. See also [8].

We always suppose $K$ to be of positive characteristic $p$, and we prove the following result:

Theorem. Let $\alpha$ be an irrational element of $K\left(\left(T^{-1}\right)\right)$. Suppose that there exist a power $q=p^{s}$ of $p$ ( $s$ integer, $s>0$ ), and polynomials $A, B, C, D$ in $K[T]$, with $A D-B C \neq 0$, such that $\alpha=\left(A \alpha^{q}+B\right) /\left(C \alpha^{q}+D\right)$. Then $\nu(\alpha)$ is a rational number, and $B(\alpha, \nu(\alpha)) \neq 0, \neq \infty$.

Of course, this result is also true when $q=1$ and $C \neq 0$, for then $\alpha$ is quadratic. Hence we suppose $q>1$.

It was already proved by J. F. Voloch ([8]) that for such an algebraic element $\alpha$, one has $B(\alpha, \nu(\alpha)) \neq 0$, but this result is also a direct consequence of the proof of the above theorem.

Let us remark that every algebraic element $\alpha$ in $K\left(\left(T^{-1}\right)\right)$ of degree 3 over $K(T)$ satisfies an equation as in the Theorem. One can take $q=p$, since the elements $1, \alpha, \alpha^{p}, \alpha^{p+1}$ are linearly dependent over $K(T)$.

All the examples of algebraic irrational elements in $K\left(\left(T^{-1}\right)\right)$ for which the value of $\nu(\alpha)$ is known satisfy an equation as in the Theorem. Nevertheless, there exist algebraic irrational elements which do not satisfy any equation of this type. For instance, let $f(X)$ be the following polynomial over $K(T)$ :

$$
f(X)=X^{p^{2}}+T^{2} X^{p}-T^{2} X+T .
$$

This polynomial is irreducible over $K(T)$, since it is a $T$-Eisenstein polynomial. It has $p$ roots in $K\left(\left(T^{-1}\right)\right)$ since the polynomial $T^{-2} f(X)$ becomes $X^{p}-X$ in the residue class field $K$. A root $\alpha$ of $f(X)$ in $K\left(\left(T^{-1}\right)\right)$ may not satisfy an equation of the type $\alpha=\left(A \alpha^{q}+B\right) /\left(C \alpha^{q}+D\right)$. Indeed, there exist polynomials $A_{s}, B_{s}, C_{s}$ in $K[T]$, for each $s \geq 0$, with

$$
\alpha^{p^{s}}=A_{s} \alpha^{p}+B_{s} \alpha+C_{s}
$$


and, by induction, it is easily seen that for $s \geq 2$,

$$
\operatorname{deg}\left(A_{s}\right)=\operatorname{deg}\left(B_{s}\right)=2\left(p^{s-1}-1\right) /(p-1) .
$$

Hence $A_{s} \neq 0$ for every $s \geq 1$. But

$$
\alpha^{p^{s}+1}=A_{s} \alpha^{p+1}+B_{s} \alpha^{2}+C_{s} \alpha
$$

and the elements $1, \alpha, \alpha^{2}, \ldots, \alpha^{p+1}$ are linearly independent over $K(T)$. So the elements $\alpha^{p^{s}+1}, \alpha^{p^{s}}, \alpha, 1$ are linearly independent over $K(T)$ for every $s \geq 1$.

To prove the Theorem, we will construct chains of rational approximations of $\alpha$ in the following way: starting from a rational approximation $P_{0} / Q_{0}$, we take

$$
P_{1} / Q_{1}=\left(A P_{0}^{q}+B Q_{0}^{q}\right) /\left(C P_{0}^{q}+D Q_{0}^{q}\right),
$$

and then we iterate the process. Let $P_{n}^{\prime}, Q_{n}^{\prime}$ be relatively prime polynomials in $K[T]$ such that $P_{n}^{\prime} / Q_{n}^{\prime}=P_{n} / Q_{n}$. A critical point is to calculate $\operatorname{deg}\left(Q_{n}^{\prime}\right)$. The next section is devoted to this.

\section{Iterated sequences. Admissible equations}

LEMMA 1. Let $E$ be a complete field of positive characteristic $p$, with a discrete valuation. Suppose that the residue class field $K$ of $E$ is a finitely generated extension of $\mathbb{F}_{p}$. Let $A, B, C$ and $D$ be elements of $E$ such that $A D-B C \neq 0$. Let $q=p^{s}$ with $s$ integer, $s>0$. Set $E^{\prime}=E \cup\{\infty\}$, and consider the map $\varphi: E^{\prime} \rightarrow E^{\prime}$, defined by $\varphi(z)=\left(A z^{q}+B\right) /\left(C z^{q}+D\right)$. There exists an integer $h>0$ (depending only upon $\varphi$ ) such that for every sequence $\left(u_{n}\right)_{n \in \mathbb{N}}$ in $E^{\prime}$ for which $u_{n}=\varphi\left(u_{n-1}\right)$ for each $n \geq 1$, either the sequence $\left(u_{h n}\right)_{n \in \mathbb{N}}$ is convergent in $E^{\prime}$, or for each $a \in E$, the sequence $\left(\left|u_{n}-a\right|\right)_{n \in \mathbb{N}}$ is constant, except for two values of $n$ at most. (The last eventuality is possible only when $K$ is infinite.)

Proof. Let us begin with the particular case $C=0$, i.e. $\varphi$ of the type $\varphi(z)=a_{1} z^{q}+b_{1}$, where $a_{1}, b_{1}$ are elements of $E, a_{1} \neq 0$. Since we may replace $E$ by an extension of finite degree, we can suppose that there exists $z_{1} \in E$ such that $\varphi\left(z_{1}\right)=z_{1}$. Define $\phi(z)=a_{1} z^{q}$. Then $\varphi(z)-z_{1}=\phi\left(z-z_{1}\right)$ for every $z \in E^{\prime}$, thus the sequence $u_{n}^{\prime}=u_{n}-z_{1}$ satisfies $u_{n}^{\prime}=\phi\left(u_{n-1}^{\prime}\right)$ for $n \geq 1$. We can furthermore suppose that there exists $z_{2} \in E$ such that $z_{2}^{1-q}=a_{1}$. Then the sequence $u_{n}^{\prime \prime}=u_{n}^{\prime} / z_{2}$ satisfies $u_{n}^{\prime \prime}=\left(u_{n-1}^{\prime \prime}\right)^{q}$. Accordingly, we have $u_{n}^{\prime \prime}=\left(u_{0}^{\prime \prime}\right)^{q^{n}}$ for each $n$. Hence if $\left|u_{0}^{\prime \prime}\right|<1$, we have $\lim u_{n}^{\prime \prime}=0$; if $\left|u_{0}^{\prime \prime}\right|>1$, we have $\lim u_{n}^{\prime \prime}=\infty$. Now suppose $\left|u_{0}^{\prime \prime}\right|=1$. Since the residue class field $K$ is finitely generated, the set of the elements of $K$ algebraic over $\mathbb{F}_{p}$ is a finite extension $\mathbb{F}_{r}$ of $\mathbb{F}_{p}$. Let $h$ be a positive integer such that $\mathbb{F}_{r} \subset \mathbb{F}_{q^{h}}$ (one can take for $h$ the degree of $\mathbb{F}_{r}$ over $\mathbb{F}_{p}$ ). Denote by 
$\overline{u_{n}^{\prime \prime}}$ the image of $u_{n}^{\prime \prime}$ in $K$. If $\overline{u_{0}^{\prime \prime}}$ is algebraic over $\mathbb{F}_{p}$, we have $\left(\overline{u_{0}^{\prime \prime}}\right)^{q^{h}}=\overline{u_{0}^{\prime \prime}}$.

That means that

$$
\left|\left(u_{0}^{\prime \prime}\right)^{q^{h}}-u_{0}^{\prime \prime}\right|<1
$$

Since

$$
\left|\left(u_{0}^{\prime \prime}\right)^{q^{h(n+1)}}-\left(u_{0}^{\prime \prime}\right)^{q^{h n}}\right|=\left|\left(u_{0}^{\prime \prime}\right)^{q^{h}}-u_{0}^{\prime \prime}\right|^{q^{h n}},
$$

the sequence $\left(u_{h n}^{\prime \prime}\right)_{n \in \mathbb{N}}$ is convergent in $E$. Finally, if $\overline{u_{0}^{\prime \prime}}$ is transcendental over $\mathbb{F}_{p}$, we have $\left(\overline{u_{0}^{\prime \prime}}\right)^{k} \neq\left(\overline{u_{0}^{\prime \prime}}\right)^{j}$ for each pair $(k, j)$ of distinct integers. Let $b \in E$. If $|b| \neq 1$, we have $\left|u_{n}^{\prime \prime}-b\right|=\max (|b|, 1)$ for every $n \in \mathbb{N}$; if $|b|=1$, let $\bar{b}$ be the residue class of $b(\bar{b} \in K)$. There exists at most one integer $n \geq 0$ such that $\left(\overline{u_{0}^{\prime \prime}}\right)^{q^{n}}=\bar{b}$, so $\left|u_{n}^{\prime \prime}-b\right|=1$ for every integer $n \geq 0$, except possibly for one value of $n$. Accordingly the sequence $\left(u_{n}\right)$ satisfies the same condition, i.e. either the sequence $\left(u_{h n}\right)_{n \in \mathbb{N}}$ is convergent in $E^{\prime}$ or, for each $a \in E,\left|u_{n}-a\right|$ is constant except for one value of $n$, at most.

In the general case $\varphi(z)=\left(A z^{q}+B\right) /\left(C z^{q}+D\right)$, we can suppose that there exists $z_{0} \in E$ such that $\varphi\left(z_{0}\right)=z_{0}$. Then there exists a function $\psi$ of the previous form such that $1 /\left(\varphi(z)-z_{0}\right)=\psi\left(1 /\left(z-z_{0}\right)\right)$. Hence there exists $h>0$ (depending only upon $\varphi$ ) such that, if we set $v_{n}=1 /\left(u_{n}-z_{0}\right.$ ), then either $\left(v_{h n}\right)_{n \in \mathbb{N}}$ is convergent in $E^{\prime}$, or $\left|v_{n}-b\right|$ is constant except for at most one value of $n$, for each $b \in E$. Thus either $\left(u_{h n}\right)_{n \in \mathbb{N}}$ is convergent in $E^{\prime}$, or $\left|u_{n}-a\right|$ is constant except for two values of $n$ at most, for each $a \in E$. Indeed,

$$
\left|u_{n}-a\right|=\left|1 / v_{n}+z_{0}-a\right|=\left|\left(1+\left(z_{0}-a\right) v_{n}\right) / v_{n}\right|,
$$

and the sequences $\left(\left|v_{n}\right|\right)$ and $\left(\left|v_{n}+1 /\left(z_{0}-a\right)\right|\right)$, when $a \neq z_{0}$, are both constant, except for two values of $n$ at most.

COROLlary. Let $E$ be a field of positive characteristic $p$, with a discrete valuation. Suppose that the residue class field $K$ of $E$ is a finitely generated extension of $\mathbb{F}_{p}$. Let $A, B, C$ and $D$ be elements of $E$ such that $A D-B C$ $\neq 0$. Let $q=p^{s}$ where $s$ is a positive integer. Denote by $\varphi$ the map from $E^{\prime}=E \cup\{\infty\}$ into $E^{\prime}$, defined by $\varphi(z)=\left(A z^{q}+B\right) /\left(C z^{q}+D\right)$. For any positive integer $h$, define $\varphi^{h}=\varphi \circ \ldots \circ \varphi$ ( $h$ times $)$. Then there exist coefficients $A_{h}, B_{h}, C_{h}, D_{h}$ in $E$, with $A_{h} D_{h}-B_{h} C_{h} \neq 0$, such that

$$
\varphi^{h}(z)=\left(A_{h} z^{q^{h}}+B_{h}\right) /\left(C_{h} z^{q^{h}}+D_{h}\right) .
$$

There is a positive integer $h$ such that for every sequence $\left(u_{n}\right)_{n \in \mathbb{N}}$ in $E \backslash\{0\}$ such that $u_{n}=\varphi\left(u_{n-1}\right)$ for each $n \geq 1$ the sequences $\left(\left|C_{h} u_{h n}^{q^{h}}+D_{h}\right|\right)_{n \in \mathbb{N}}$ and $\left(\left|A_{h}+B_{h} / u_{h n}^{q^{h}}\right|\right)_{n \in \mathbb{N}}$ have both a constant finite positive value when $n$ is large. 
Proof. The form of $\varphi$ is clear. We take the matrices

$$
M_{h}=\left[\begin{array}{ll}
A_{h} & B_{h} \\
C_{h} & D_{h}
\end{array}\right]
$$

satisfying

We have

$$
M_{h}=M_{h-1}\left[\begin{array}{ll}
A^{q^{h-1}} & B^{q^{h-1}} \\
C^{q^{h-1}} & D^{q^{h-1}}
\end{array}\right]
$$

$$
A_{h} D_{h}-B_{h} C_{h}=\operatorname{det} M_{h}=(A D-B C)^{\left(q^{h}-1\right) /(q-1)} .
$$

Since we may replace $E$ by its completion, we can suppose that $E$ is complete. We choose $h$ just as in Lemma 1 ; then we can suppose that $h=1$. If the sequence $\left(u_{n}\right)_{n \in \mathbb{N}}$ is convergent in $E^{\prime}$, then $\left|C u_{n}^{q}+D\right|$ is constant for $n$ large. Indeed, let $\beta=\lim u_{n}$. If $\beta=\infty$, one has $C=0$, for $\varphi(\infty)=\infty$, and the result is trivial. If $\beta \neq \infty$, the sequence $\left(C u_{n}^{q}+D\right)$ is convergent in $E$ to the limit $C \beta^{q}+D \neq 0$, for $\varphi(\beta)=\beta$. Hence $\left|C u_{n}^{q}+D\right|=\left|C \beta^{q}+D\right|$ when $n$ is large. One sees in a similar way that $\left|A+B / u_{n}^{q}\right|$ is constant for large $n$. If now the sequence $\left(u_{n}\right)$ is not convergent in $E^{\prime}$, then $\left|u_{n}-a\right|$ is constant except for two values of $n$ at most. Clearly the same is true for $\left|C u_{n}^{q}+D\right|$ and for $\left|A+B / u_{n}^{q}\right|$.

We can now define an admissible equation. We return to the Theorem: let $\alpha$ be an element of $K\left(\left(T^{-1}\right)\right)$ satisfying $\alpha=\left(A \alpha^{q}+B\right) /\left(C \alpha^{q}+D\right)$ where $A, B, C, D$ are polynomials in $K[T]$ with $A D-B C \neq 0$. Let $\Pi$ be an irreducible polynomial in $K[T]$. We will use the $\Pi$-adic absolute value on $K(T)$, which is defined by $|\Pi|_{\Pi}=1 /|\Pi|$ and $|f|_{\Pi}=1$ if $f$ is a polynomial not divisible by $\Pi$. Consider the map $\varphi$ of the set $K\left(\left(T^{-1}\right)\right) \cup\{\infty\}$ into itself defined by $\varphi(z)=\left(A z^{q}+B\right) /\left(C z^{q}+D\right)$. When $\Pi$ is an irreducible polynomial dividing $A D-B C$, we say that $\alpha=\varphi(\alpha)$ is a $\Pi$-admissible equation for $\alpha$ if the Corollary of Lemma 1 holds with $h=1$ for the field $K(T)$ with the $\Pi$-adic absolute value. Clearly, in proving the Theorem, we may suppose that $K$ is finitely generated over $\mathbb{F}_{p}$; then so is the residue class field of $K(T)$ for the $\Pi$-adic absolute value. Then the Corollary of Lemma 1 applies, and it is clear from the proof that there exists a positive integer $h_{\Pi}$ such that the Corollary holds for every multiple $h$ of $h_{\Pi}$. Hence the equation $\alpha=\varphi^{h}(\alpha)$ is $\Pi$-admissible for every multiple $h$ of $h_{\Pi}$. We say that the equation $\alpha=\varphi(\alpha)$ is admissible if it is $\Pi$-admissible for each irreducible polynomial $\Pi$ dividing $A D-B C$. Now, there does exist an admissible equation for $\alpha$. Indeed, there is only a finite number of irreducible divisors of $A D-B C$, and so if $h$ is a common multiple of the integers $h_{\Pi}$ when $\Pi$ divides $A D-B C$, the equation $\alpha=\varphi^{h}(\alpha)$ is admissible (since $A_{h}, B_{h}, C_{h}, D_{h}$ are polynomials such that $A_{h} D_{h}-B_{h} C_{h}$ is a power of $A D-B C$, it follows that $A_{h} D_{h}-B_{h} C_{h}$ and $A D-B C$ have the same irreducible divisors). 
ExAmples. For $\alpha \in K\left(\left(T^{-1}\right)\right)$ such that $\alpha^{q-1}=D / A$, where $A, D$ are relatively prime polynomials such that $|A|=|D|>1$, the equation $\alpha=$ $A \alpha^{q} / D$ is trivially admissible. Baum and Sweet's equation $\alpha=T /\left(T \alpha^{2}+1\right)$ over $\mathbb{F}_{2}(T)$ is admissible. So is also Taussat's equation $\alpha=T /\left(T \alpha^{3}+1\right)$ over $\mathbb{F}_{3}(T)$. But over $\mathbb{F}_{2}(T)$, the equation $\alpha^{3}=D / A$, where $A, D$ are relatively prime polynomials such that $|A|=|D|>1$, has the form $\alpha=D /\left(A \alpha^{2}\right)$, which is not admissible.

\section{Chains of convergents}

LEMma 2. Let $\alpha$ be an irrational element of $K\left(\left(T^{-1}\right)\right)$ satisfying an equation $\alpha=\left(A \alpha^{q}+B\right) /\left(C \alpha^{q}+D\right)$, where $A, B, C, D$ are polynomials in $K[T]$ such that $A D-B C \neq 0$, and $q=p^{s}$ (where $s$ is a positive integer). Let $P, Q$ be polynomials in $K[T], Q \neq 0$. Define

$$
R=A P^{q}+B Q^{q}, \quad S=C P^{q}+D Q^{q} .
$$

Assume that

$$
|\alpha-P / Q|<\left|\alpha^{q}+D / C\right|^{1 / q} \quad \text { if } C \neq 0
$$

(there is no condition if $C=0$ ). Then $S \neq 0$ and

$$
|\alpha-R / S|=|A D-B C|\left|C \alpha^{q}+D\right|^{-2}|\alpha-P / Q|^{q} .
$$

If furthermore we have

$$
|\alpha-P / Q|<|A D-B C|^{-1 /(q-1)}\left|C \alpha^{q}+D\right|^{2 /(q-1)}
$$

and

$$
|\alpha-P / Q|<|A D-B C|^{-1 /(q-1)} /|Q|^{2}
$$

then the polynomials $R$ and $S$ satisfy conditions (i), (ii), (iii). The rational fractions $P / Q, R / S$, are convergents of $\alpha$ (in the continued fraction expansion). If $P^{\prime}, Q^{\prime}, R^{\prime}, S^{\prime}$ are polynomials in $K[T]$ such that $\left(P^{\prime}, Q^{\prime}\right)=$ $\left(R^{\prime}, S^{\prime}\right)=1$ and $P / Q=P^{\prime} / Q^{\prime}, R / S=R^{\prime} / S^{\prime}$, then $\left|S^{\prime}\right|>\left|Q^{\prime}\right|$.

Proof. First notice that $\left|C(P / Q)^{q}+D\right|=\left|C \alpha^{q}+D\right|$ by (i). Hence $|S|=\left|C \alpha^{q}+D \| Q\right|^{q}>0$. Now we write

$$
\alpha-R / S=\left(A \alpha^{q}+B\right) /\left(C \alpha^{q}+D\right)-\left(A P^{q}+B Q^{q}\right) /\left(C P^{q}+D Q^{q}\right)
$$

hence

$$
|\alpha-R / S|=|A D-B C|\left|C \alpha^{q}+D\right|^{-2}|\alpha-P / Q|^{q} .
$$

Define $\varepsilon$ and $\eta$ by

$$
|\alpha-P / Q|=\varepsilon /|Q|^{2}, \quad|\alpha-R / S|=\eta /|S|^{2} .
$$

We have $\eta=|A D-B C| \varepsilon^{q}$, hence $\eta<\varepsilon$ by (iii). By (ii) we have $|\alpha-R / S|<$ $|\alpha-P / Q|$. Thus conditions (i), (ii), (iii) are satisfied by the couple $(R, S)$. 
Since $\eta<\varepsilon<1, P / Q$ and $R / S$ are convergents of $\alpha$, and $\left|S^{\prime}\right|>\left|Q^{\prime}\right|$ as $|\alpha-R / S|<|\alpha-P / Q|$.

The conditions of Lemma 2 are hereditary, so we can iterate the process. But even if $P$ and $Q$ are relatively prime, $R$ and $S$ are not necessarily so. In order to calculate the degree of their gcd, we have to use an admissible equation for $\alpha$.

LEMMA 3. With the notations of Lemma 2, assume moreover that the equation $\alpha=\left(A \alpha^{q}+B\right) /\left(C \alpha^{q}+D\right)$ is admissible. Let $P$ and $Q$ be relatively prime polynomials in $K[T], Q \neq 0$. Assume that the couple $(P, Q)$ satisfies the conditions (i), (ii), (iii) of Lemma 2. We define sequences of polynomials $\left(P_{n}\right)_{n \in \mathbb{N}}$ and $\left(Q_{n}\right)_{n \in \mathbb{N}}$ by

$$
P_{0}=P, \quad Q_{0}=Q,
$$

and for $n \geq 1$ :

$$
P_{n}=A P_{n-1}^{q}+B Q_{n-1}^{q}, \quad Q_{n}=C P_{n-1}^{q}+D Q_{n-1}^{q} .
$$

Then $Q_{n} \neq 0$ for each $n$. Let $P_{n}^{\prime}$ and $Q_{n}^{\prime}$ be relatively prime polynomials such that $P_{n} / Q_{n}=P_{n}^{\prime} / Q_{n}^{\prime}$. There exist real constants $C_{1}>0, C_{2}, \delta>1, \lambda>0$ such that $\operatorname{deg}\left(Q_{n}^{\prime}\right)=C_{1} q^{n}+C_{2}$ and $\left|Q_{n}^{\prime}\right|^{\delta}\left\|Q_{n}^{\prime} \alpha\right\|=\lambda$ for all sufficiently large $n$. One has $0<C_{1} \leq \operatorname{deg}(Q)+m /(q-1)$ where $\left|C \alpha^{q}+D\right|=|T|^{m}$. Moreover, $\delta$ is a rational number.

Proof. It is clear that the couple $\left(P_{n}, Q_{n}\right)$ satisfies conditions (i), (ii), (iii) in Lemma 2, for each $n$. Hence $Q_{n} \neq 0$. Set $\operatorname{deg}\left(\alpha-P_{n} / Q_{n}\right)=-r_{n}$ and $\operatorname{deg}(A D-B C)=c$. By Lemma 2, we have $r_{n}=q r_{n-1}+2 m-c$ for every $n \geq 1$, thus

$$
r_{n}=\left(r_{0}+(2 m-c) /(q-1)\right) q^{n}-(2 m-c) /(q-1) \quad \text { for all } n .
$$

We are now going to calculate $\operatorname{deg}\left(Q_{n}^{\prime}\right)$. First we calculate $\operatorname{deg}\left(Q_{n}\right)$. Since

$$
\left|Q_{n}\right|=\left|C \alpha^{q}+D\right|\left|Q_{n-1}\right|^{q},
$$

we have

$$
\operatorname{deg}\left(Q_{n}\right)=q \operatorname{deg}\left(Q_{n-1}\right)+m
$$

hence

$$
\operatorname{deg}\left(Q_{n}\right)=(\operatorname{deg}(Q)+m /(q-1)) q^{n}-m /(q-1) .
$$

We are going to prove that we also have $\operatorname{deg}\left(Q_{n}^{\prime}\right)=C_{1} q^{n}+C_{2}$ for all large $n$. It suffices to prove an analogous form for the degree of the (monic) $\operatorname{gcd} D_{n}=\left(P_{n}, Q_{n}\right)$ :

$$
\operatorname{deg}\left(D_{n}\right)=C_{3} q^{n}+C_{4} \quad\left(C_{3}, C_{4} \text { real constants }\right) .
$$

As $\left(P_{0}, Q_{0}\right)=1, P_{n}$ and $Q_{n}$ have no other common irreducible divisors than the irreducible divisors of $A D-B C$. It suffices to calculate $\left|D_{n}\right|_{\Pi}$ for each element $\Pi$ of the finite set of the irreducible divisors of $A D-B C$. 
Denote by $w_{\Pi}$ the $\Pi$-adic valuation on $K(T)$ such that $|f|_{\Pi}=|\Pi|^{-w_{\Pi}(f)}$ for all $f \in K(T), f \neq 0$. Now it is clear that it suffices to prove that for each irreducible divisor $\Pi$ of $A D-B C$, there exist real constants $F, F^{\prime}, G, G^{\prime}$ (depending upon $\Pi$ ) such that $w_{\Pi}\left(P_{n}\right)=F q^{n}+F^{\prime}$ and $w_{\Pi}\left(Q_{n}\right)=G q^{n}+G^{\prime}$ when $n$ is large.

We write $Q_{n}=\left(C\left(P_{n-1} / Q_{n-1}\right)^{q}+D\right) Q_{n-1}^{q}$. As the equation $\alpha=$ $\left(A \alpha^{q}+B\right) /\left(C \alpha^{q}+D\right)$ is admissible, $\left|C\left(P_{n-1} / Q_{n-1}\right)^{q}+D\right|_{\Pi}$ is constant when $n$ is large. Thus there exists a real constant $b$ such that $w_{\Pi}\left(Q_{n}\right)=$ $q w_{\Pi}\left(Q_{n-1}\right)+b$ for all large $n$. So we have $w_{\Pi}\left(Q_{n}\right)=G q^{n}+G^{\prime}$ where $G, G^{\prime}$ are real constants, for all large $n$. We can proceed in the same way to compute $w_{\Pi}\left(P_{n}\right)$, as $P_{n} \neq 0$ for all large $n$. Indeed, in $K\left(\left(T^{-1}\right)\right)$ we have $\lim P_{n} / Q_{n}=\alpha$. Then we can write $P_{n}=\left(A+B\left(Q_{n-1} / P_{n-1}\right)^{q}\right) P_{n-1}^{q}$ and apply the Corollary of Lemma 1.

Thus $\operatorname{deg}\left(Q_{n}^{\prime}\right)=C_{1} q^{n}+C_{2}$ when $n$ is large. As $\lim \operatorname{deg}\left(Q_{n}^{\prime}\right)=+\infty$, we have $C_{1}>0$. Moreover, $C_{1} \leq \operatorname{deg}(Q)+m /(q-1)$, for $\operatorname{deg}\left(Q_{n}^{\prime}\right) \leq \operatorname{deg}\left(Q_{n}\right)$. Now, let $\delta=\left(r_{0}+(2 m-c) /(q-1)\right) / C_{1}-1$. Then $(\delta+1) \operatorname{deg}\left(Q_{n}^{\prime}\right)-r_{n}$ is constant when $n$ is large. Hence $\left|Q_{n}^{\prime}\right|^{\delta}\left\|Q_{n}^{\prime} \alpha\right\|$ is a positive constant when $n$ is large. We have $\delta>1$, for $C_{1} \leq \operatorname{deg}(Q)+m /(q-1)<\left(r_{0}+(2 m-c) /(q-1)\right) / 2$ by (iii). Clearly $C_{1}$ is a rational number, accordingly so is $\delta$.

Now we fix an admissible equation $\alpha=\left(A \alpha^{q}+B\right) /\left(C \alpha^{q}+D\right)$ for $\alpha$, and we call a sequence $\left(P_{n}^{\prime} / Q_{n}^{\prime}\right)_{n \in \mathbb{N}}$ of rational approximations of $\alpha$ as in Lemma 3 a chain of convergents of $\alpha$. That means that the couple $\left(P_{0}^{\prime}, Q_{0}^{\prime}\right)$ satisfies the conditions (i), (ii), (iii) of Lemma 2, and that

$$
P_{n}^{\prime} / Q_{n}^{\prime}=\left(A P_{n-1}^{\prime q}+B Q_{n-1}^{\prime q}\right) /\left(C P_{n-1}^{\prime q}+D Q_{n-1}^{\prime q}\right) \quad \text { for each } n \geq 1 .
$$

For such a chain $\mathcal{C}=\left(P_{n}^{\prime} / Q_{n}^{\prime}\right)_{n \in \mathbb{N}}$, with relatively prime polynomials $P_{n}^{\prime}$ and $Q_{n}^{\prime}$ for each $n$, it follows from Lemma 3 that there exists a rational constant $\delta>1$ such that $\left|Q_{n}^{\prime}\right|^{\delta}\left\|Q_{n}^{\prime} \alpha\right\|$ is constant when $n$ is large. Then we say that $\mathcal{C}$ is a $\delta$-chain. As the sequence $\left(\operatorname{deg}\left(Q_{n}^{\prime}\right)\right)$ is strictly increasing, every chain is included in a maximal chain, that is to say, a chain $\left(P_{n}^{\prime \prime} / Q_{n}^{\prime \prime}\right)_{n \in \mathbb{N}}$ for which there exists no rational fraction $P_{-1}^{\prime \prime} / Q_{-1}^{\prime \prime}$ such that $\left(P_{n}^{\prime \prime} / Q_{n}^{\prime \prime}\right)_{n \geq-1}$ is a chain. Since the map $z \mapsto\left(A z^{q}+B\right) /\left(C z^{q}+D\right)$ is injective, any two chains are either disjoint, or one is included in the other. Every chain including a $\delta$-chain is also a $\delta$-chain. For any chain $\mathcal{C}$ we denote by $\delta(\mathcal{C})$ the constant $\delta$ such that $\mathcal{C}$ is a $\delta$-chain.

LEMmA 4. Let $\delta_{0}$ be a real number, $\delta_{0}>1$. There exist only a finite number of maximal chains $\mathcal{C}$ of convergents of $\alpha$ with $\delta(\mathcal{C}) \geq \delta_{0}$.

Proof. Distinct maximal chains are disjoint. We will prove that if we have $N$ disjoint chains $\mathcal{C}_{k}(1 \leq k \leq N)$, with $\delta\left(\mathcal{C}_{k}\right) \geq \delta_{0}$, then $\delta_{0}^{N-1}<$ $q$. Define $\mathcal{C}_{k}=\left(P_{n, k} / Q_{n, k}\right)_{n \in \mathbb{N}}$ where $P_{n, k}$ and $Q_{n, k}$ are relatively prime polynomials. For each $k$ there exist real constants $C_{k}>0$ and $C_{k}^{\prime}$ such 
that $\operatorname{deg}\left(Q_{n, k}\right)=C_{k} q^{n}+C_{k}^{\prime}$ for all sufficiently large $n$. We can modify the indexation by replacing $n$ by $n+n_{k}$ for each $k$, where $n_{k}$ is an integer in $\mathbb{Z}$, so that we get $1 \leq C_{k}<q$. Notice that for $k \neq j$, the couples $\left(C_{k}, C_{k}^{\prime}\right)$ and $\left(C_{j}, C_{j}^{\prime}\right)$ are distinct. Indeed, $\operatorname{deg}\left(Q_{n, k}\right) \neq \operatorname{deg}\left(Q_{n, j}\right)$ for each $n$, since $\mathcal{C}_{k}$ and $\mathcal{C}_{j}$ are disjoint. Thus, we can suppose that for each integer $k$ such that $1 \leq k<N$, we have $C_{k}<C_{k+1}$ or $C_{k}=C_{k+1}$ and $C_{k}^{\prime}<C_{k+1}^{\prime}$. If $Q$ is the denominator of a convergent of $\alpha$, let $Q^{*}$ be the denominator of the next convergent. One has $\|Q \alpha\|=1 /\left|Q^{*}\right|([3])$. Accordingly, as $\mathcal{C}_{k}$ is a chain with $\delta\left(\mathcal{C}_{k}\right) \geq \delta_{0}$, there exists a constant $\sigma$ such that

$$
\operatorname{deg}\left(Q_{n, k}^{*}\right) \geq \delta_{0} \operatorname{deg}\left(Q_{n, k}\right)-\sigma .
$$

Since, for any integer $k$ such that $1 \leq k<N$, we have $\operatorname{deg}\left(Q_{n, k+1}\right)>$ $\operatorname{deg}\left(Q_{n, k}\right)$ when $n$ is large, thus we have

$$
\operatorname{deg}\left(Q_{n, k+1}\right) \geq \delta_{0} \operatorname{deg}\left(Q_{n, k}\right)-\sigma \quad \text { for all large } n \text {. }
$$

Therefore

$$
\lim _{n \rightarrow \infty} \operatorname{deg}\left(Q_{n, k+1}\right) / \operatorname{deg}\left(Q_{n, k}\right)=C_{k+1} / C_{k} \geq \delta_{0} .
$$

Hence we conclude that $\delta_{0}^{N-1}<q$. One can notice, with a similar proof, that we even have $\delta_{0}^{N} \leq q$.

4. Proof of the Theorem. The result is obvious if $B(\alpha, 1) \neq 0$ (thus $\nu(\alpha)=1)$. If $B(\alpha, 1)=0$, it follows from Lemma 3 that there exist chains of convergents of $\alpha$ (we have fixed an admissible equation for $\alpha$ ). By Lemma 4 , the numbers $\delta(\mathcal{C})$, where $\mathcal{C}$ runs over the set of chains of $\alpha$, achieve a maximum $\delta$. For every $\delta$-chain $\mathcal{C}$, denote by $\lambda(\mathcal{C})$ the (constant) value of $|Q|^{\delta}\|Q \alpha\|$ when $P / Q \in \mathcal{C}$, with polynomials $P, Q$ relatively prime, and $\operatorname{deg}(Q)$ large. Since there exist only a finite number of maximal $\delta$-chains (when $\delta$ is maximal) we can define $\Lambda$ as being the minimum of $\lambda(\mathcal{C})$ for all the $\delta$-chains $\mathcal{C}$. Clearly $\Lambda$ is finite, but not zero. We are going to prove that $B(\alpha, \delta)=\Lambda$. That will show that $\nu(\alpha)=\delta$, and the Theorem will be proved.

Let $\left(P_{n} / Q_{n}\right)$ be a sequence of convergents of $\alpha$, with relatively prime polynomials $P_{n}, Q_{n}$. We suppose that $\lim \left|Q_{n}\right|=+\infty$, and that the sequence $\left(\left|Q_{n}\right|^{\delta}\left\|Q_{n} \alpha\right\|\right)$ is bounded. We must prove that for all large $n$, $P_{n} / Q_{n}$ belongs to the union of the $\delta$-chains. But by Lemma $3, P_{n} / Q_{n}$ is the first term of a chain for all large $n$. This chain is a $\nu_{n}$-chain, with $\nu_{n}=\left(\varrho_{n}+(2 m-c) /(q-1)\right) / C_{n}-1$, where $\varrho_{n}=-\operatorname{deg}\left(\alpha-P_{n} / Q_{n}\right)$ and $0<$ $C_{n} \leq \operatorname{deg}\left(Q_{n}\right)+m /(q-1)$ (see Lemma 3$)$. Since the sequence $\left(\left|Q_{n}\right|^{\delta}\left\|Q_{n} \alpha\right\|\right)$ is bounded, there exists a real constant $\tau$ such that $(\delta+1) \operatorname{deg}\left(Q_{n}\right)-\varrho_{n} \leq-\tau$, and thus

$$
\nu_{n} \geq\left(\delta \operatorname{deg}\left(Q_{n}\right)+\tau+(m-c) /(q-1)\right) /\left(\operatorname{deg}\left(Q_{n}\right)+m /(q-1)\right) .
$$


Hence $\lim \nu_{n}=\delta$. Then we conclude by Lemma 4 that $\nu_{n}=\delta$ for all large $n$, so $P_{n} / Q_{n}$ belongs to the union of the $\delta$-chains. Hence it is clear that $B(\alpha, \delta)=\Lambda$.

5. Examples. We can now treat examples. We consider the case of an equation $X^{e}=R$, where $e$ is a positive integer, not divisible by $p$, and $R \in K(T)$. Such an equation has a root in $K\left(\left(T^{-1}\right)\right.$ ) if (and only if ) $\operatorname{deg}(R)$ is a multiple of $e$ and the first coefficient of $R$ belongs to $K^{e}$. There exists a positive integer $s$ such that $e$ divides $p^{s}-1$ (we can take for $s$ the order of $p$ in the multiplicative group $\left.(\mathbb{Z} / e \mathbb{Z})^{*}\right)$. Therefore if an element $\alpha \in K\left(\left(T^{-1}\right)\right)$ is a root of an equation $\alpha^{e}=R$, with $R \in K(T)$, it also satisfies an equation $\alpha^{q-1}=R^{\prime}$, with $q=p^{s}$ and $R^{\prime} \in K(T)$. We can write this equation as $\alpha=A \alpha^{q} / D$ where $A, D$ are polynomials such that $R^{\prime}=D / A$. Accordingly, if $\alpha \notin K(T)$, our result applies. Notice that the equation $\alpha=A \alpha^{q} / D$ is trivially admissible.

We give explicit calculations in the case $p=2, e=3$. We prove:

Corollary. Let $\alpha, \alpha^{\prime}, \alpha^{\prime \prime}$ be elements of $\mathbb{F}_{2}\left(\left(T^{-1}\right)\right)$ such that $\alpha^{3}=$ $\left(T^{3}+T+1\right) / T^{3}, \alpha^{\prime 3}=\left(T^{4}+T^{2}+T+1\right) / T^{4}, \alpha^{\prime \prime 3}=\left(T^{4}+T+1\right) / T^{4}$. One has: $\nu(\alpha)=3 / 2, B(\alpha, 3 / 2)=1 ; \nu\left(\alpha^{\prime}\right)=4 / 3, B\left(\alpha^{\prime}, 4 / 3\right)=1 ; \nu\left(\alpha^{\prime \prime}\right)=5 / 4$, $B\left(\alpha^{\prime \prime}, 5 / 4\right)=|T|^{-3}$.

Pr o of. The first terms of the expansion of $\alpha$ in continued fraction are:

$$
\alpha=1+1 \sqrt{T^{2}}+T+1 \sqrt{T}+1+1 \sqrt{\ldots}
$$

The first convergents are $P_{0} / Q_{0}=1, P_{1} / Q_{1}=\left(T^{2}+T+1\right) /\left(T^{2}+T\right)$, and $\left|\alpha-P_{1} / Q_{1}\right|=|T|^{-5}$.

We start from the convergent $P_{1} / Q_{1}$, and we construct by Lemma 2 the sequence of convergents $\left(P_{n, 1} / Q_{n, 1}\right)_{n \in \mathbb{N}}$ :

$$
P_{n, 1} / Q_{n, 1}=\left(T^{2}+T+1\right)^{4^{n}} /\left((T+1)^{4^{n}} T\left(T^{3}+T+1\right)^{\left(4^{n}-1\right) / 3}\right),
$$

which is the sequence of rational (irreducible) fractions obtained from the relations $P_{0,1} / Q_{0,1}=P_{1} / Q_{1}$ and, for $n \geq 1$,

$$
P_{n, 1} / Q_{n, 1}=\left(T^{3} /\left(T^{3}+T+1\right)\right)\left(P_{n-1,1} / Q_{n-1,1}\right)^{4} \text {. }
$$

Since $\left|\alpha-P_{1} / Q_{1}\right|=|T|^{-5}$, we have for each $n,\left|\alpha-P_{n, 1} / Q_{n, 1}\right|=|T|^{-5 \cdot 4^{n}}$. We have $\operatorname{deg}\left(Q_{n, 1}\right)=2 \cdot 4^{n}$, hence $\operatorname{deg}\left(Q_{n, 1}^{*}\right)=3 \cdot 4^{n}$. The sequence $\left(P_{n, 1} / Q_{n, 1}\right)$ is a $3 / 2$-chain (for $n \geq 1$ ).

Now we notice that if we write the equation for $\alpha$ in the (non-admissible) form $\alpha=D / A \alpha^{2}$ (with $D=T^{3}+T+1$ and $A=T^{3}$ ), we see by Lemma 2(i) that we can deduce from an approximation $P / Q$ of $\alpha$, with $|\alpha-P / Q|<1$, the approximation $D Q^{2} / A P^{2}$. We have $\left|\alpha-D Q^{2} / A P^{2}\right|=|\alpha-P / Q|^{2}$. Hence for $n \geq 1$, we obtain from $P_{n, 1} / Q_{n, 1}$ the convergent

$$
P_{n, 2} / Q_{n, 2}=(T+1)^{2 \cdot 4^{n}}\left(T^{3}+T+1\right)^{\left(2 \cdot 4^{n}+1\right) / 3} / T\left(T^{2}+T+1\right)^{2 \cdot 4^{n}} .
$$


We have $\left|\alpha-P_{n, 2} / Q_{n, 2}\right|=|T|^{-10 \cdot 4^{n}}$ and $\operatorname{deg}\left(Q_{n, 2}\right)=4^{n+1}+1$ (of course $P_{n, 2}$ and $Q_{n, 2}$ are relatively prime). Accordingly $\operatorname{deg}\left(Q_{n, 2}^{*}\right)=6 \cdot 4^{n}-1$. The sequence $\left(P_{n, 2} / Q_{n, 2}\right)_{n \geq 1}$ is another $3 / 2$-chain. There is no other maximal $\delta$ chain, with $\delta \geq 3 / 2$, than $\left(P_{n, 1} / Q_{n, 1}\right)$ and $\left(P_{n, 2} / Q_{n, 2}\right)$, with $n \geq 1$. Indeed, for each denominator of a convergent $Q$ of $\alpha$ such that $2 \cdot 4^{n} \leq \operatorname{deg}(Q)<$ $2 \cdot 4^{n+1}$, with $n \geq 1$, if $Q \neq Q_{n, 1}$ and $Q \neq Q_{n, 2}$, then $\operatorname{deg}(Q)$ and $\operatorname{deg}\left(Q^{*}\right)$ both belong to one of the intervals $\left[3 \cdot 4^{n}, 4^{n+1}+1\right]$ or $\left[6 \cdot 4^{n}-1,2 \cdot 4^{n+1}\right]$, hence it is clear that any other maximal chain is a $\delta$-chain with $\delta \leq 4 / 3$. Therefore we have $\nu(\alpha)=3 / 2$. Since $\left|Q_{n, 1}\right|^{3 / 2}\left\|Q_{n, 1} \alpha\right\|=1$ and $\left|Q_{n, 2}\right|^{3 / 2}\left\|Q_{n, 2} \alpha\right\|=$ $|T|^{5 / 2}$ for each $n \geq 1$, we have $B(\alpha, 3 / 2)=1$. It is easy to see that the inequality $|Q|^{3 / 2}\|Q \alpha\| \geq 1$ holds for any polynomial $Q$ of degree $>3$.

For $\alpha^{\prime}$ and $\alpha^{\prime \prime}$, we only indicate sufficient chains of convergents; we give the degrees of the denominators of these convergents and of the next convergent.

For $\alpha^{\prime}$ :

$$
\begin{gathered}
\left(3 \cdot 4^{n}, 4^{n+1}\right), \quad\left(6 \cdot 4^{n}, 8 \cdot 4^{n}\right) \quad(n \geq 0) \\
\left((4 / 3)\left(4^{n+2}-1\right),(4 / 3)\left(17 \cdot 4^{n}+1\right)\right) \quad(n \geq 0) \\
\left((4 / 3)\left(2 \cdot 4^{n+2}+1\right),(4 / 3)\left(34 \cdot 4^{n}-1\right)\right) \quad(n \geq 1) .
\end{gathered}
$$

For $\alpha^{\prime \prime}$ :

$$
\begin{array}{cc}
\left((4 / 3)\left(4^{n}-1\right),\left(5 \cdot 4^{n}+4\right) / 3\right) & (n \geq 0) \\
\left((4 / 3)\left(8 \cdot 4^{n}+1\right),(4 / 3)\left(10 \cdot 4^{n}-1\right)\right) & (n \geq 1), \\
\left(2 \cdot 4^{n+1}, 9 \cdot 4^{n}\right), \quad\left(4^{n+2}, 18 \cdot 4^{n}\right) \quad(n \geq 0) .
\end{array}
$$

6. Open problems. We know nothing (except the Liouville theorem) about the approximation exponent of algebraic elements $\alpha$ which do not satisfy any non-trivial equation of the form $\alpha=\left(A \alpha^{q}+B\right) /\left(C \alpha^{q}+D\right)$, where $q$ is a power of the characteristic $p \neq 0$ of $K$. One can ask if Roth's theorem $\nu(\alpha)=1$ holds for these elements. For instance, it is possible to calculate, by computer - I thank Y. Taussat - many terms of the expansion in continued fraction of both the roots in $\mathbb{F}_{2}\left(\left(T^{-1}\right)\right)$ of the equation $X^{4}+$ $T^{2} X^{2}+T^{2} X+T=0$ (see $\S 1$ ). It seems that for a root $\alpha$ of this equation, one has $\nu(\alpha)=1$ (but $B(\alpha, 1)=0)$.

For the algebraic elements satisfying a non-trivial equation

$$
\alpha=\left(A \alpha^{q}+B\right) /\left(C \alpha^{q}+D\right),
$$

there are examples with $\nu(\alpha)=1$ (see [1], [4]). But no criterion is known. For instance, we do not know whether for an irrational element $\alpha$ of $K\left(\left(T^{-1}\right)\right)$ such that there exists a positive integer $e$ with $\alpha^{e} \in K(T)$, one may have $\nu(\alpha)=1$ (when $\alpha$ is not quadratic). 


\section{References}

[1] L. E. Baum and M. M. Sweet, Continued fractions of algebraic power series in characteristic 2, Ann. of Math. 103 (1976), 593-610.

[2] A. Blanchard et M. Mendès-France, Symétrie et transcendance, Bull. Sci. Math. 106 (3) (1982), 325-335.

[3] B. de Mathan, Approximations diophantiennes dans un corps local, Bull. Soc. Math. France. Mém. 21 (1970).

[4] W. H. Mills and D. P. Robbins, Continued fractions for certain algebraic power series, J. Number Theory 23 (1986), 388-404.

[5] C. F. Osgood, Effective bounds on the "diophantine approximation" of algebraic functions over fields of arbitrary characteristic and applications to differential equations, Indag. Math. 37 (1975), 105-119.

[6] Y. Taussat, Approximations diophantiennes dans un corps de séries formelles, Thèse de 3ème cycle, Bordeaux, 1986.

[7] S. Uchiyama, On the Thue-Siegel-Roth theorem III, Proc. Japan Acad. 36 (1960), $1-2$.

[8] J. F. Voloch, Diophantine approximation in positive characteristic, Period. Math. Hungar. 19 (3) (1988), 217-225.

UNIVERSITÉ DE BORDEAUX 1, MATHÉMATIQUES

(AND U.A. C.N.R.S. 226)

351 COURS DE LA LIBÉRATION

F-33405 TALENCE CEDEX, FRANCE 